\title{
Gobernanza universitaria: enfoques y alcances conceptuales
}

\author{
Adrián Acosta-Silva, Francisco Ganga-Contreras y Claudio Rama-Vitale
}

\section{RESUMEN}

Estudiar las relaciones entre gobernanza, políticas públicas y desempeño institucional en el campo de la educación superior, se ha transformado en un gran reto para los investigadores, pero también en una atractiva oportunidad, dada la complejidad que esta triada de conceptos representa. El trabajo tiene como propósito realizar una reflexión de carácter teórico respecto de los alcances conceptuales que tiene la gobernanza universitaria y sus vínculos con las políticas públicas y su correspondiente desempeño, con la finalidad de precisar la terminología y sus alcances más significativos para América Latina. Se trata de una investigación descriptiva, de carácter documental, basada en la exploración de la bibliografía sociológica y politológica contemporánea.

Palabras clave: educación superior, gobernanza universitaria, desempeño institucional, complejidad, régimen de políticas, América Latina.

Adrián Acosta-Silva

aacosta@cucea.udg.mx Mexicano. Doctor en Investigación en Ciencias Sociales con especialización en Ciencia Política, Flacso-México, Sociólogo, Universidad de Guadalajara, México. Profesor-investigador titular C, Instituto de Investigaciones en Políticas Públicas y Gobierno, CUCEA-Universidad de Guadalajara, México. Temas de investigación: políticas públicas y cambios institucionales en educación superior; gobernanza institucional y desempeño universitario; poder y autonomía universitaria en América Latina. ORCID: https://orcid.org/0000-0003-2312-8990. Universidad de Los Lagos, Chile. Consultor Internacional, Profesor Titular del Departamento de Educación de la Facultad de Educación y Humanidades de la Universidad de Tarapacá, Chile. Temas de investigación: gobernanza universitaria, desempeño universitario, liderazgo, administración de organizaciones. ORCID: https://orcid.org/0000-0001-9325-6459. 


\section{Governança universitária: enfoques e alcances conceituais}

\section{RESUMO}

Estudar as relações entre governança, políticas públicas e desempenho institucional no campo da educação superior, tem se transformado em um grande desafio para os pesquisadores, mas também em uma atrativa oportunidade, dada a complexidade que esta tríade de conceitos representa. $\mathrm{O}$ trabalho tem como propósito realizar uma reflexão de caráter teórico sobre os alcances conceituais que tem a governança universitária e seus vínculos com as políticas públicas e seu correspondente desempenho, com a finalidade de definir a terminologia e seus alcances mais significativos para América Latina. Se trata de uma pesquisa descritiva, de carácter documental, baseada na exploração da bibliografia sociológica e de ciências políticas contemporânea.

Palavras chave: educação superior, governança universitária, desempenho institucional, complexidade, regime de políticas, América Latina.

\section{University governance: conceptual approaches and extents}

\section{ABSTRACT}

Studying the relationships between governance, public policy and institutional performance in the field of higher education has become a great challenge for researchers, but also an attractive opportunity, given the complexity that this triad of concepts represents. The purpose of this work is to carry out a theoretical reflection on the conceptual scope of university governance and its connection with public policy and its corresponding performance, in order to specify the terminology and its most relevant significance for Latin America. This research is descriptive and documentary, based on the exploration of the contemporary sociological and political literature.

Key words: higher education, university governance, institutional performance, complexity, policy regime, Latin America. 


\section{Introducción}

Las relaciones entre políticas públicas, gobernanza y desempeño institucional en el campo de la educación superior suelen ser empíricamente difusas. No hay una relación directa, causal, entre estos componentes de la acción pública en la explicación, descripción y análisis de los problemas del gobierno, la eficacia directiva, o el impacto de la productividad académica de las organizaciones de educación superior en su desempeño institucional. Ello no obstante, en los últimos años ha despuntado un renovado interés en varias disciplinas de las ciencias sociales por identificar la "compejidad causal" de esas relaciones, un interés a veces orientado a producir conocimiento sobre los distintos factores de esos componentes relacionales o, en otros casos, con una marcada intención hacia la construcción de modelos de gobernanza para la resolución de problemas prácticos de la gestión y el cambio institucional en la educación superior.

Paradójicamente, la difusa complejidad causal de la gobernanza institucional de la educación superior coexiste con un espíritu de época dominado abrumadoramente por la "épica de los indicadores" (Acosta, 2019). Nuevas exigencias de calidad y competitividad institucional están en el centro de la multiplicación de las ofertas institucionales dirigidas hacia la productividad académica, la internacionalización, la eficiencia administrativa, el aseguramiento de la calidad, el mejoramiento de los aprendizajes a través de la incorporación de nuevas tecnologías de información y comunicación, o el impulso a la investigación científica, el desarrollo tecnológico y la innovación en las universidades. Las recompensas puestas en juego son claras: prestigio, reputación, legitimidad. Este entorno de exigencias y demandas configura la agenda del gobierno de la educación superior contemporánea, una agenda que atrae a nuevos actores universitarios y no universitarios, y explica parcialmente la expansión acelerada de la educación superior pública y privada en prácticamente todo el mundo.
En el centro de esta agenda está el tema de la gobernanza universitaria. Su origen, sus modelos o formas, sus prácticas y estructuras, sus actores, tensiones y conflictos, así como su articulación y pertinencia con las diversas tipologías universitarias, habitan la agenda de investigación que se ha desarrollado con distinta intensidad entre las diversas comunidades epistémicas y comunidades de políticas interesadas en la comprensión o resolución de los problemas del gobierno de la educación superior. Gobernanza implica cooperación y coordinación, legitimidad y estabilidad, arreglos institucionales y actores estratégicos, políticas institucionales y acción pública.

El artículo es un esfuerzo por identificar y distinguir cuatro tipos de relaciones fundamentales: 1) las de la gobernanza universitaria con las políticas públicas; 2) los desplazamientos conceptuales tradicionales y la "invención" de un nuevo mapa analítico; 3) el problema del desempeño institucional, y 4) la relación entre los indicadores de la gobernanza con los indicadores del desempeño. A través de la exploración de la bibliografía sociológica y politológica contemporánea, el texto intenta ofrecer un panorama actualizado de los principales autores, enfoques y conceptos que alimentan el desarrollo del estudio de la gobernanza universitaria contemporánea.

Junto con precisar la terminología y las implicancias más significativas de la gobernanza en las universidades de América Latina, también se busca contribuir a organizar y profundizar la discusión académica sobre el tema desde una perspectiva latinoamericana, con la intención de producir nuevas preguntas de investigación a partir de los hallazgos acumulados en las últimas décadas.

\section{Gobernanza, desempeño y políticas públicas}

Desde el inicio del siglo XXI se instaló firmemente en la agenda nacional e internacional de las reformas de la educación superior el tema de la gobernanza 
institucional, pero se debe partir reconociendo que este concepto está en proceso de consolidación y en la actualidad se encuentra en plena etapa de discusión y afianzamiento (Ganga et al., 2014). De hecho, aún no es factible encontrar una definición concordada y universalmente aceptada por la comunidad científica. En general, la expresión apunta al ejercicio de control colectivo para el logro de fines comunes; en un sentido más extenso, podría afirmarse que es un concepto omniabarcante, referido a un proceso de dirección y a la gestión de las disposiciones que los actores públicos y/o privados tienen para resolver los problemas sociales, vertebrando las metas de los diversos grupos de interés en búsqueda de alcanzar objetivos colectivos valorados públicamente (Boer y File, 2009; Li et al., 2019; Aguilar, 2007; Ganga-Contreras y Nuñez, 2018; Ganga-Contreras et al., 2017; Brunner et al., 2018).

Contextualizado en las organizaciones, los aportes investigativos de Erwin, Landry, Livingston y Dias (2019), basados principalmente en lo planteado por Hambrick, Werder y Zajac (2008), concluyen, desde una perspectiva internalista, que cualquier modelo de gobernanza debe tener en cuenta la estructura formal (composición de los directorios), la estructura conductual (problemas de poder entre los actores claves) y el comportamiento de los procesos (procedimientos que pueden dificultar o mejorar la efectividad de los directorios y el impacto de los factores externos en los procesos).

Pero hay factores contextuales decisivos en la comprensión de los cambios en las gobernanzas universitarias contemporáneas, Por distintas razones intelectuales, políticas y de políticas públicas, el énfasis en la gestión de las reformas se asoció de manera implícita al gobierno de las transformaciones impulsadas por la configuración de un entorno de políticas de educación superior basadas en un paradigma que combina institucionalización de la evaluación, tipologías universitarias, rendición de cuentas (accountability), operación de esquemas de acreditación y aseguramiento de la calidad, impulso a la diversificación de la oferta y la demanda pública y privada, promoción de la internacionalización, y combinación del financiamiento público/ gubernamental condicionado, competitivo y diferenciado con un papel creciente del financiamiento privado, auto-generado por las propias universidades (Shattock, 2014; Amaral et al., 2002; Brown, 2011; Teichler, 1998). En su conjunto, estas políticas generales están orientadas a la construcción de "universidades de clase mundial", centradas en la investigación, competitivas, prestigiadas, atractivas para lo que se preveía como un contexto de internacionalización creciente de la educación terciaria en todo el mundo (Altbach et al., 2011).

Las causas profundas de ese giro paradigmático tienen que ver con una crisis de legitimidad de las universidades públicas entre las nuevas élites dirigentes. Esa legitimidad se tradujo en dos fenómenos principales: la desconfianza y el escepticismo sobre el desempeño de las universidades públicas (Zgaga, 2012). Esas actitudes están en la base de los diagnósticos que desde finales del siglo $\mathrm{XX}$ asociaron los problemas del financiamiento público con la baja calidad y la ineficiencia e ineficacia de la educación superior universitaria. Bajo este paradigma general, las políticas de educación superior impulsadas por los gobiernos nacionales siguieron una ruta de transformaciones que favorecieron un nuevo modo de gestión institucional de las políticas y los recursos asociados a ellas. Dos modelos "clásicos" de gobernanza universitaria se distinguen bajo la influencia de ese paradigma: el "modelo de planeación y control racional" de naturaleza normativa y centralizada, y el "modelo de auto-regulación", basado en la descentralización de las decisiones y de los recursos (Van Vught et al., 2015). Mediante diversos instrumentos y fórmulas, la gobernanza se constituyó como sinónimo de implementación de las políticas, justamente como ocurrió en otros campos de la acción pública (Capano et al., 2017; Cairney, 2018). En otros casos, la gobernanza se 
articuló como un enfoque centrado en el análisis de la capacidad y la eficacia directiva de los gobiernos nacionales, o los gobiernos corporativos tanto públicos como privados (Broucker et al., 2015; Aguilar, 2007, 2018). A través de diversos esquemas de incentivos, estímulos y recompensas a los desempeños individuales, grupales y organizacionales, articulados a distintos "paquetes" de restricciones y constreñimientos simbólicos, normativos e institucionales, las universidades experimentaron los efectos de nuevas reglas asociadas a entornos políticos y de políticas cualitativamente distintos a los que permitieron la primera ola de masificación y expansión de los años sesenta y setenta del siglo pasado (Acosta, 2012a).

Las nuevas condiciones (las políticas públicas) influyeron en una transformación significativa del perfil y funciones del gobierno universitario. Las tradiciones asentadas en las estructuras y prácticas del co-gobierno universitario operando en un esquema de autonomía "fuerte" respecto de las fuerzas del Estado y del mercado, y que estaban orientadas por la lógica del aseguramiento de la legitimidad, la estabilidad y la eficacia de las universidades públicas, fueron explicaciones insuficientes cuando las reglas del juego pasaron de las intervenciones "suaves" del gobierno hacia intervenciones basadas en el "control" o "timoneo a distancia", orientadas a asegurar la producción de indicadores comparables de desempeño del sistema de educación superior y, específicamente, de las universidades públicas. Esas nuevas reglas reconfiguraron la gobernanza de la educación superior desde un modo colegiado hacia un modo "corporativo" o "juntista" (Boardism), definido como "el declive del poder de los académicos en los procesos de hechura de las decisiones universitarias", y el creciente "rol de los interesados externos (external stakeholders) en el gobierno de las instituciones de educación superior" (Veiga et al., 2015: 400).
La centralización de la dirección de las universidades mediante la conformación de un núcleo directivo especializado significó la disminución del papel de los académicos y de los estudiantes en la conducción de los asuntos institucionales; es decir, las tradiciones del gobierno compartido (shared governance) en las universidades fueron transformadas deliberadamente por la irrupción de una tendencia hacia la conformación de una burocracia profesional dedicada exclusivamente a la gestión de los cambios en los patrones de coordinación y conducción de la universidad, orientados hacia el mejoramiento de la eficiencia y la eficacia directiva universitaria (Clark, 1998; Birnbaum, 2004; Bowen et al., 2015). Otras perspectivas han tratado de asociar los cambios en la dirección de las universidades hacia modelos que se mueven entre la "democratización colegiada" y la "centralización del poder", o entre la "personalización política" y el "gerenciamiento modernizador" (Atairo et al., 2015).

En lo que existe coincidencia general es que la crisis de las fórmulas tradicionales del financiamiento público experimentada desde la década de los ochenta del siglo pasado se convirtió en el "mecanismo de disparo" de las nuevas políticas educativas de nivel terciario, que significó la construcción de nuevos arreglos institucionales entre las universidades, el Estado y el mercado. En no pocos casos nacionales, las políticas basadas en incentivos se expresaron en múltiples instrumentos de financiamiento público extraordinario, competitivo, condicionado y diferencial, que configuraron nuevas agendas de cambio institucional en las propias universidades y sistemas de educación superior. ${ }^{1}$

El triángulo de hierro de la coordinación de los sistemas que propuso Clark (1983), se transformó en un esquema útil pero insuficiente para explicar las nuevas reglas y relaciones de la cooperación y coordinación . al..nivel..de las .instituciones . de ..educación

\footnotetext{
${ }^{1}$ El impacto de las políticas de incentivos en el desempeño de la educación superior se expresa de manera diferente en los países latinoamericanos debido a las distintas configuraciones de los sistemas nacionales de educación terciaria. Ello no obstante, es posible afirmar que esas políticas modificaron significativamente los esquemas de gobernanza institucional, en especial, de las universidades públicas (de Vries y Álvarez-Mendiola, 2012; Acosta, 2020).
} 
superior (Maggio, 2011). Sobre esos paradigmas de regulación de mercado, Estado y academia, irrumpieron nuevos agregados de actores destacándose lo internacional y la sociedad civil, haciendo modelos de regulación más complejos (Rama, 2015).

El análisis de la coordinación de los sistemas cedió el paso al análisis del gobierno institucional como foco o unidad central para comprender cómo se gobiernan las universidades en un entorno de cambios y multiplicación de los actores interesados en la educación superior (Musselin y Mignot-Gérard, 2002; El-Khawas, 2002). Más aún: el estudio de los modos y experiencias de los gobiernos universitarios, y las maneras en que concilian o equilibran sus estructuras de gobierno, gobernabilidad y gobernanza, se convirtió en una perspectiva novedosa para tratar de explicar las decisiones, estructuras, actores y procesos gubernativos de las universidades públicas. También irrumpieron como parte de la diferenciación de tipologías, dinámicas de gobernabilidad y gobernanza asociadas a los comportamientos institucionales universitarios (Brunner, 2011).

\section{El doble desplazamiento y la "invención" de un nuevo mapa analítico}

$\mathrm{Al}$ inicio del siglo XXI la experiencia de tensiones, conflictos y cambios en las relaciones de las universidades con sus entornos requería de nuevos anteojos conceptuales y analíticos para comprender el papel del gobierno universitario en el desempeño institucional. A la luz de dichas experiencias, un nuevo esfuerzo intelectual se desarrolló sobre la base de un doble desplazamiento analítico. Por un lado, el desplazamiento de las teorías clásicas del gobierno universitario como ejercicio de racionalización y administración institucional. Del otro lado, un desplazamiento de enfoques de la gobernabilidad basados en el análisis de la representación y la participación hacia los enfoques de la gobernanza basados en la capacidad institucional y la eficacia directiva. Ello daría por resultado el descubrimiento, o "reinvención", de un horizonte reflexivo nuevo: el de las relaciones entre gobernanza y desempeño institucional (Tight, 2015; King, 2015).

Para el caso latinoamericano, la transición del énfasis de la gobernabilidad hacia el énfasis en la gobernanza está intensamente relacionado con los cambios contextuales e institucionales de la educación superior. Los numerosos ensayos, estudios y ejercicios en torno al fenómeno del co-gobierno universitario, que se centraron en los relatos sobre las diversas formas de participación y representación de los principales actores de la universidad en sus esquemas de gobierno, se orientaron hacia las explicaciones en torno a las relaciones del co-gobierno con la autonomía académica y la legitimidad de las prácticas políticas universitarias (Atairo, 2016). Pero con la emergencia de cambios sociopolíticos contextuales (democratización de los regímenes políticos), y bajo la influencia de nuevos paradigmas de políticas en la educación superior (rendición de cuentas, evaluación de la calidad y financiamiento público diferenciado, condicionado y competitivo), el foco del análisis se desplazó hacia el proceso mismo de gobernar de las universidades, donde la eficacia directiva para adaptarse a las nuevas condiciones y tensiones contextuales se colocó en el centro de las esfuerzos comprensivos sobre el tema del gobierno universitario (Acosta, 2012b). En la región, también la rápida expansión y diferenciación institucional producida desde los años setenta, fue derivando en la aparición de nuevas formas de gobierno más pertinente y con ello de nuevas tipologías institucionales de universidades, todo lo cual derivó en nuevos marcos legales de legitimación y reconocimiento de otras formas de organización institucional y distribución de los espacios de poder.

Ello explica la irrupción del concepto de gobernanza en el campo universitario. Aunque existen varias definiciones del concepto, la idea de la gobernanza se impuso poco a poco en el terreno de las interpretaciones orientadas hacia la solución de los problemas más allá de su utilización en la comprensión de los 
problemas asociados a tipos de gobernanza institucionales. De algún modo, la gobernanza se colocó como la lente conceptual principal en la búsqueda de la coordinación y cooperación entre diversos actores para identificar objetivos y estrategias comunes orientadas hacia la gestión del cambio institucional, entendido básicamente como el proceso de adaptación de los sistemas e instituciones de educación a las transformaciones ocurridas en sus entornos institucionales, regionales y globales. De este modo, los problemas clásicos del poder, la autoridad y el gobierno de la educación superior fueron reinterpretados a través de los cristales y anteojos de la gobernanza (Reale et al., 2015; Rodríguez, 2014).

Ese giro interpretativo constituyó una novedad importante en el campo del análisis latinoamericano. El análisis de la gestión del cambio (la gobernanza) sustituyó al análisis de la gestión del conflicto (gobernabilidad) (Acosta, 2018). La experiencia del gobierno compartido en las universidades, centradas en la búsqueda de legitimidad y la eficacia de la autoridad, fueron desplazadas o subordinadas a las prioridades de la gobernanza institucional centradas en la búsqueda del prestigio académico, la calidad institucional y la eficiencia de la gestión (Bowen y Tobin, 2015; Bernasconi y Clasing, 2015). La expansión de las ofertas y demandas de la educación superior, la búsqueda de la calidad, la diversificación y diferenciación institucional, los cambios en las relaciones entre lo público y lo privado, la continua mezcla de diversos instrumentos de políticas que combinan estímulos financieros y recompensas simbólicas para la promoción de cambios en las instituciones y sistemas, se colocaron en el centro de la acción pública.

Importa considerar el contexto en el cual se impulsó la perspectiva de la gobernanza como el eje de las reformas de la educación superior. Una compleja mixtura de ideas generales e intereses específicos se conjugaron para formular una perspectiva de acción pública, que colocó el acento en los principios de la gestión y coordinación sistémica e institucional de las intervenciones gubernamentales en varios campos de políticas (y no sólo los relacionados con la educación universitaria), que modificaron y flexibilizaron las propias estructuras del Estado (Hardiman, 2012; Pierre, 2012). La lenta irrupción de nuevos sistemas de educación superior con flexibilidad, sistemas de créditos, posgrados, sistemas de aseguramiento de la calidad, pasantías, sedes y virtualización fueron marcando nuevas formas de la gobernanza y la gestión institucional (Rama, 2014). De manera silenciosa pero sistemática, el lenguaje de la gobernanza ha dominado la configuración de los cambios institucionales, y buena parte de los esfuerzos y prácticas universitarias se comenzaron a justificar como expresiones de mejoramiento de las gobernanzas institucionales y sistémicas: calidad, eficiencia, cobertura, competitividad, equidad, evaluación.

El supuesto de este lenguaje es que la gobernanza está relacionada con el desempeño o rendimiento del sistema y de las instituciones de educación superior, así como con sus estructuras. De entrada, tendría que advertirse la existencia de distintos tipos de gobernanza que se relacionan con distintos tipos de funciones y de desempeño en el campo de la educación superior universitaria. Ello conduce a la diferenciación analítica entre los regímenes de gobernanza (governance regimes), los regímenes de políticas (policy regimes) y los regímenes de calidad (quality regimes). El primero distingue a los sistemas de gobernanza como esquemas generales de identificación de las relaciones entre las instituciones universitarias y sus entornos y actores. Un ejemplo es el propuesto por Brunner (2011) al ofrecer una tipología de regímenes de gobernanza sistémica en la educación superior latinoamericana: "emprendurista" (o de mercado), "político", "colegiado", o de "partes interesadas" (stake-holders).

Por su parte, el enfoque del régimen de políticas se orienta hacia la caracterización de los factores que influyen en el rendimiento del sistema: el contexto institucional, el entorno de políticas, los actores 
involucrados, los usos y costumbres de las organizaciones, y los sistemas de creencias, son fuerzas que determinan significativamente los comportamientos institucionales que producen las políticas públicas, y que permiten explicar las complejas relaciones entre gobernanza y desempeño (Wilson, 2000). El aporte analítico de este enfoque consiste en la identificación de los factores determinantes o condicionantes del comportamiento institucional que producen de manera intencional, deliberada, las políticas públicas en el campo de la educación superior universitaria.

A su vez, el enfoque de regímenes de calidad se orienta a la identificación de los factores que determinan la calidad del desempeño institucional, a partir del análisis de las relaciones en dos ejes: "Atención a la reputación" y "Atención a la excelencia", distinguiendo entre regímenes "venerables", "excelsos", "misioneros" o "aspirantes" (Paradeise y Thoenig, 2017).

Estos tres enfoques permiten apreciar con mayor nitidez la naturaleza y complejidad causal de las relaciones entre gobernanza y desempeño universitario. Pero resulta particularmente apropiado el enfoque de régimen de políticas para producir un conocimiento más preciso de las causas y los efectos del gobierno, los procesos de gobierno y el análisis del desempeño institucional de las universidades latinoamericanas. Definidos como "arreglos de gobierno para abordar problemas de políticas públicas", la perspectiva de regímenes "hace hincapié en la interacción de las ideas, los arreglos institucionales y los actores interesados que subyacen a un régimen determinado" (May, 2018: 284). Esa perspectiva ayuda a "identificar las realidades de cómo se aborda un conjunto dado de problemas y las dinámicas políticas que suscitan esas realidades"(loc. cit.).

\section{El problema del desempeño institucional}

Pero al lado de las dificultades conceptuales y métricas de la gobernanza universitaria se encuentra también la conceptualización del desempeño o rendimiento institucional (institutional performance). Ese concepto debe a economistas como Douglass North (1993) y a politólogos como Easton (1985) o Putnam (1993), un acercamiento analítico como el conjunto de resultados (outcomes) que se desprenden de la eficacia y eficiencia con la cual las organizaciones (jugadores) se adaptan exitosamente a las instituciones (reglas del juego). Desde el punto de vista sociológico, autores como Lascoumes y Le Galès (2014), han destacado el análisis del impacto de las políticas públicas en el desempeño de las instituciones y los grupos. En estos casos, el marco institucional juega un papel decisivo para que las sociedades, las empresas privadas, o las organizaciones sociales, políticas o educativas, obtengan buenos resultados en términos de eficiencia o crecimiento económico, confianza, credibilidad y legitimidad política, o en la percepción de un rendimiento exitoso o satisfactorio en términos de las expectativas sociales o gubernamentales depositadas en ellas.

Desde el punto de vista del neoinstitucionalismo económico, North (1993) señala que el factor clave que explica un buen desempeño es la determinación del costo de transacción de los intercambios entre las organizaciones y los individuos. Ese costo se determina fundamentalmente por la información que está disponible para los "equipos" (las organizaciones) involucrados en cada campo de acción específico, y son las reglas institucionales las que pueden reducir o incrementar los costos de acceso a dicha información. Desde esta perspectiva, el desempeño está asociado a los costos de transacción y a la información disponible. Un desempeño eficiente será el resultado de bajos costos de transacción en un marco institucional que provea reglas claras y comunes para el acceso a la información indispensable para la toma de las decisiones de las organizaciones.

Desde el punto de vista de la sociología de la acción pública y de la ciencia política, el desempeño institucional se asocia con el grado de legitimidad y confianza que se percibe entre las comunidades y sociedades en las cuales se encuentran insertas. En el primer caso (la sociología de la acción pública), 
Lascoumes y Le Galés (2014: 19-20) proponen un modelo de análisis a partir de la identificación de cinco variables relacionadas entre sí: actores (individuales o colectivos), representaciones (marcos cognitivos y normativos), instituciones (marcos de acción), procesos (movilizaciones de los actores) y resultados (efectos sobre las organizaciones e impactos sobre los problemas públicos que se desea resolver). Desde la ciencia política, Robert Putnam (1993), por ejemplo, desde el neoinstitucionalismo político, al definir a las instituciones como "reglas de acción" y como fuentes de creación de capital social (confianza), muestra que el incremento de la confianza reduce los costos de transacción y mejora el desempeño de las propias instituciones. Autores como Pierre Rosanvallon (2007: 23), por su parte, señalan que la confianza "constituye una especie de institución invisible" que cumple con una función estratégica, como "economizador institucional" que permite ahorros en términos de "evaluación, verificación y prueba".

La OECD (2002: 29) en su "Glosario" de los principales términos sobre evaluación y gestión basada en resultados, define desempeño como "el grado al cual una intervención pública o un actor del desarrollo opera de acuerdo a ciertos criterios/normas/ directrices o logra resultados de acuerdo a los planes establecidos". Así, los "Indicadores de desempeño", son concebidos como "una variable cuantitativa o cualitativa que permite verificar los cambios generados por una intervención pública, relativa a lo que estaba planeado".

Swianiewicz (2000) analiza el desempeño desde el punto de vista de los resultados finales o impacto de la acción de la entidad en el cumplimiento de su misión, y también de cómo éstos fueron logrados en términos de la eficiencia (uso de recursos para la generación de los productos), eficacia (capacidad de cumplir con la producción comprometida y de logro de objetivos), calidad (capacidad de lograr la producción de los bienes y servicios de acuerdo con los atributos esperados por los usuarios), y economía (cómo se organizaron los recursos económicos y presupuestarios para el logro de los resultados) (Swianiewicz, 2000; CEPAL, 2005).

El problema del desempeño aparece en el campo de la educación superior como el resultado de intervenciones del Estado o del mercado como "proveedores" de reglas de acción, a través de la configuración de un régimen de políticas más o menos estable. Para el caso de las universidades públicas latinoamericanas, es el gobierno federal, central o nacional, a partir del diseño e implementación de políticas públicas, el marco de referencia para la orientación del desempeño institucional esperado (Kent, 2009; Acosta, 2010). La legitimidad del desempeño institucional es el resultado de la eficacia de sus resultados y procesos. No es sólo una creencia colectivamente compartida, normativamente determinada de arriba hacia abajo (Top-Down), o gobernada mediante instrumentos como el financiamiento público y estímulos para el comportamiento de las universidades, sino que se articula estrechamente a la confianza sobre la eficacia institucional de sus procedimientos y estructuras.

\section{Relación entre los indicadores de la gobernanza y los indicadores del desempeño}

Los indicadores de la gobernanza como combinatoria de poder y estructura, están determinados por los fines institucionales y por el mercado específico donde se actúa, que impone sus particularidades en términos de la oferta y de sus características.

Existe una correlación entre la diferenciación institucional, la diversificación académica, la expansión de la cobertura, los niveles de calidad y los tipos de gobernanza. En este sentido la vinculación entre la diversidad de las universidades y la gobernanza, ha planteado la necesidad de formular y analizar diversas tipologías universitarias para facilitar la comprensión de los sistemas de educación y la eficiencia de los modelos de gobernanza. 
Uno de los primeros aportes metodológicos fue, desde la década de los sesenta, la clasificación de las instituciones de educación superior propuesto por la Fundación Carnegie (McCormick, 2013), que concibió un sistema altamente diversificado. A partir de esta tipología inicial, se han formulado otras clasificaciones de universidades atendiendo a las distintas zonas geográficas, como la European Classification of Higher Education Institutions, o a casos nacionales como son los que se han realizado en Alemania, Brasil, España, Francia, México, Reino Unido y Estados Unidos (Ganga et al., 2017). Muchas legislaciones nacionales han realizado sus propias clasificaciones de universidades.

Robert Birnbaum (1983) consideró distintas metodologías para analizar la diversidad de los sistemas de educación superior y construyó un aparato conceptual, con diversas dimensiones de análisis, que permiten alimentar múltiples tipologías y clasificaciones. Es un debate que se ha incrementado en los últimos años como resultado de la irrupción de los distintos rankings internacionales y de las tensiones respecto a la capacidad de ellos de evaluar a las universidades en contexto de alta diversidad al interior de los sistemas universitarios a escala global. En los rankings, la utilización de menos indicadores en la evaluación hace que la selección de la tipología institucional escogida como marco referencial sea más precisa, y los rankings dominantes a escala internacional expresen más claramente un tipo de universidad centrada en la investigación de las que se ha llamado de "clase mundial" (Salmi, 2009).

Sin embargo, el problema es similar con relación a los procesos de evaluación y acreditación, que aunque tengan una mayor cantidad de factores o indicadores también contienen un marco referencial y responden a un particular modelo universitario, como eje de su enfoque de la calidad y por ende de sus indicadores de desempeño. Ello plantea la necesidad de una atención particular a criterios y enfoques diferenciados que permitan poder reconocer otros modelos de instituciones al interior de los sistemas de educación superior con sus propias modalidades de gobernanza y de resultados.

El desarrollo en varios países de la región de estándares de evaluación institucionales diferenciados atendiendo a las instituciones universitarias y no universitarias, a los distintos niveles de posgrado o a la educación a distancia, constituyen avances en la focalización de los sistemas de aseguramiento de la calidad, propendiendo a establecer criterios propios a las particularidades de los diversos subconjuntos universitarios. Sin embargo, se necesita profundizar en establecer tipologías - y por ende diferenciaciones - más ajustadas a las diversas realidades universitarias, con miras a formular cuáles son los modelos de gobernanza más eficientes para el desempeño institucional.

Es este un debate que atraviesa la región, y que se está expresando en la introducción de nuevas clasificaciones universitarias, atento a la propia complejización de los sistemas universitarios y de la diversificación de las universidades, de sus misiones y por ende de los subsectores universitarios en los cuales están insertas. Es sin duda una dinámica que se incrementa a medida que la cobertura ha ido aumentando y el sistema diversificándose.

En tanto la educación superior se ha masificado, y se ha pasado de unas pocas universidades a sistemas muy amplios de educación superior con muy diferentes instituciones universitarias, con misiones y funciones diversificadas, la discusión sobre la conceptualización de las distintas clasificaciones y tipologías ha pasado de ser un aspecto de reflexión meramente académico, a constituirse en un componente de la política pública y de las orientaciones de los sistemas de aseguramiento de la calidad. Ello en tanto la función de las tipologías y clasificaciones refiere, no a la investigación académica, sino a la ejecución de políticas públicas, especialmente del aseguramiento de la calidad, que deben estar ajustadas a las realidades y particularidades de los sistemas de educación superior. 
La focalización de la política pública constituye un basamento de los nuevos modelos de regulación de los Estados en general y de la acción pública en educación superior en particular, como área social. Focalizar la regulación y la atención no responde meramente a la búsqueda de políticas de compensación proactivas, sino a la necesidad de una delimitación más clara de los sujetos de la regulación y de las acciones en la materia. La focalización y segmentación de las políticas se basan por su parte en un conocimiento más exhaustivo y detallado de los problemas y de los instrumentos. Hernández et al., (2005), consideran que el eje de la política pública es la focalización y que ello requiere delimitar claramente subconjuntos específicos diferenciados, de acuerdo con sus problemáticas y realidades singulares.

Sistemas más amplios y diferenciados hacen más necesaria la existencia de tipologías y clasificaciones institucionales, que permiten una mayor sofisticación y particularismos de las acciones de aseguramiento de la calidad y de regulación de los mercados. McCormick (2013) ha considerado incluso que a medida que las categorías son sometidas a una definición más precisa, el número de categorías aumenta, pero al tiempo ello redunda en un grado de homogeneidad mayor al interior de dicha tipología o subconjunto y con ello políticas más focalizadas. Esto provoca que el tamaño del grupo o el peso de una categoría dentro del sistema declinen, pero que la comprensión del subsector y la focalización de la acción mejoren y con ello la propia calidad de los resultados de los procesos de aseguramiento de la calidad.

Dada la alta correlación entre la expansión de la cobertura y la diferenciación y diversificación de las instituciones, la construcción de clasificaciones se constituye en la base para el propio desarrollo de las universidades al interior de los subgrupos o cluster a través de su propia especialización de funciones y cometidos. Por lo tanto, se establece como un componente de los objetivos del desarrollo de los sistemas de educación superior y de su diversidad, la especialización de las instituciones, para alcanzar niveles superiores de desarrollo asociados a sus propias misiones, visiones e historias particulares. Ello también favorece la focalización de las políticas de regulación y de aseguramiento de la calidad.

La diversificación institucional y la propia especialización se transforman, en tal sentido, en el soporte de la calidad, al permitir responder más claramente a las demandas de algunos sectores, alcanzar curvas de experiencia superiores y focalizar los servicios y ofertas universitarias con mayor pertinencia educativa.

La función de los procesos de diferenciación y diversificación institucional no sólo está asociada a sistemas universitarios más complejos y cuyas diversidades impulsan una amplia variación de las modalidades, niveles, pedagogías, formas de gobernanza, perfiles de oferta y misiones institucionales, sino a la especialización de las instituciones dentro de subconjuntos de cluster de servicios y que al tiempo aportan a expandir el propio sistema de educación superior. Porter (2002) planteó que en los escenarios competitivos las organizaciones tienen como opciones de desarrollo, liderazgo en costos, diferenciación o focalización, con sus diferenciados niveles de desempeño y por ende indicadores, planteamientos que no se alejan mucho de la realidad del sistema universitario.

La tipología de los sistemas universitarios se constituye así, junto al tipo de gobernanza, en las variables por considerar en el análisis de los desempeños institucionales.

\section{Conclusiones}

El enfoque de la gobernanza se ha instalado firmemente en el ámbito de la reflexión y la investigación universitaria en los últimos años, como parte de procesos de diferenciación y búsqueda de alcanzar mejores logros en la eficiencia y en la gobernabilidad en contextos cada vez más competitivos y diversos. La gobernanza es uno de los nuevos centros explicativos de los conflictos en la gestión directiva, pero también se asocia a la reflexión e investigación de 
las tipologías universitarias en América Latina y El Caribe, que expresan las diversas y heterogéneas formas de ajuste de las instituciones a las condiciones del mercado y/o las demandas sociales o políticas.

Por otra parte, el concepto de desempeño tiene una fuerte legitimación en los ámbitos académicos como instrumento analítico para comprender y comparar las lógicas institucionales, complejidades y dinámicas de la educación superior y formular tipologías en este campo, que complementen y/o mejores las propuestas existentes.

En este artículo se ha argumentado que el enfoque que puede articular consistentemente el análisis de las relaciones causales entre gobernanza y desempeño

\section{Referencias}

Acosta Silva, Adrián (2020), "Autonomía universitaria y estatalidad", Revista de la Educación Superior, vol. 49, núm. 193, pp. 1-23, <http://resu.anuies.mx/ojs/index. $\mathrm{php} / \mathrm{resu} / \mathrm{article} /$ view/1025>.

Acosta Silva, Adrián (2019), "La épica de los indicadores", en Leonardo Lomelí Vanegas y Roberto Escalante Semerena (coords.), Autonomías bajo acecho, México, Siglo XXI Editores, pp. 83-97.

Acosta Silva, Adrián (2018), "Gobernanza y desempeño universitario", Revista Venezolana de Gerencia, año 23, ed. especial, núm. 1, julio, pp. 432-440, <http:// www.produccioncientifica.luz.edu.ve/index.php/rvg/ article/view/23924/24359> [Consulta: diciembre de 2019].

Acosta Silva, Adrián (2012a), “¿Tiempos líquidos? Democracia, universidad y desarrollo en México", Cuestiones de Sociología, núm. 8, <https://www. cuestionessociologia.fahce.unlp.edu.ar $>$ [Consulta: enero de 2020].

Acosta Silva, Adrián (2012b), "Federal policies and governance of universities in Mexico, 1990-2010", es el de "régimen de políticas". Como se ha expuesto aquí, ese concepto tiene una potencialidad explicativa que permite contextualizar adecuadamente los componentes históricos y coyunturales que condicionan, determinan o influyen en los cambios en los patrones de gobernanza y desempeño de las instituciones de educación superior en la región. Del mismo modo, emerge con fuerza la necesidad de ampliar estas miradas teóricas hacia investigaciones empíricas que aborden en términos concretos la situación en los países de la región. Estamos hablando, sin duda, de tópicos que abren ingentes oportunidades para continuar con indagaciones tanto teóricas como empíricas.

en Hans G. Schuetze, William Bruneau y Garnet Grosjean (eds.), University governance and reform. Policy, fads and experience in international perspective, Nueva York, Palgrave Macmillan, pp.147-160.

Acosta Silva, Adrián (2010), Príncipes, burócratas y gerentes. El gobierno de las universidades públicas en México (2a. ed.), México, ANUIES/UDUAL.

Aguilar, Luis (2007), "El aporte de la política pública y la nueva gestión pública a la gobernanza", Revista del Clad Reforma y Democracia, núm. 39, pp. 1-15.

Aguilar, Luis (2018), "La nueva gobernanza pública", en José Alberto Castellanos G., Christian M. Sánchez y Alejando Aguilar (coords.), Tendencias del gobierno y de la administración pública, Guadalajara, GUCEA, U. de G. (XXIII Congreso Internacional del CLAD sobre la Reforma del Estado y de la Administración Pública), pp. 23-41.

Aguilar, Luis (2016), Democracia, gobernabilidad y gobernanza, México, Instituto Nacional Electoral.

Altbatch, Phillip y Jamil Salmi (eds.) (2011), The road of academic excellence: the making of world-class research universities, Washington D.C., World Bank. 
Amaral, Alberto, Glen Jones y Berit Karseth (eds.) (2002), Governing higher education: national perspectives on institutional governance, Dordrecht/Boston/London, Kluwer Academic Publishers.

Atairo, Daniela (2016), El gobierno universitario en la agenda académica y política de América Latina, México, ANUIES (Col. Temas de Hoy).

Atairo, Daniela, Adrián Acosta y Antonio Camou (2015), "Gobernabilidad y democracia en las universidades públicas latinoamericanas. Argentina y México en perspectiva comparada", en Los desafios de la universidad pública en América Latina y el Caribe, Buenos Aires, CLACSO/Instituto de Investigaciones Gino Germani, Universidad de Buenos Aires, pp. 19-118.

Bernasconi, Andrés y Paula Clasing (2015), "Legitimidad en el gobierno universitario: una nueva tipología", Archivos Analíticos de Políticas Educativas, vol. 23, pp. 1-26, DOI: http://dx.doi.org/10.14507/epaa.v23.2001 [Consulta: octubre de 2019].

Birnbaum, Robert (2004), "The end of shared governance: looking ahead and looking back", en New Directions for Higher Education (Special Issue: Restructuring Shared Governance in Higher Education), vol. 2 004, núm. 127, otoño, pp. 5-22.

Birnbaum, Robert (1983), Maintaining Diversity in Higher Education, San Francisco, Jossey-Bass.

Bowen, William y Eugene Tobin (2015), Locus of authority. The Evolution of faculty roles in the governance of higher education, New Jersey, Princeton University Press.

Broucker, Bruno y Kurt de Wit (2015), "New public management in higher education", en Jerome Huisman, Harry de Boer, David D. Hill y Manuel Souto-Otero (eds.), The Palgrave International Handbook of Higher Education Policy and Governance, Basingstoke, Palgrave Macmillan, pp. 57-75.

Brown, Roger (2011), Higher education and market, Oxford, Higher Education Policy Institute.

Brunner, José Joaquín (2011), "Gobernanza universitaria, tipología, dinámicas y tendencias", Revista Educación, núm. 355, mayo-agosto, pp. 137-159.

Brunner José Joaquín, Francisco Ganga-Contreras y Emilio Rodríguez-Ponce (2018), "Gobernanza del capitalismo académico: aproximaciones desde Chile”, Revista Venezolana de Gerencia, vol. 23, núm. 1, pp. 11-35. Cairney, Paul (2018), "La implementación y el problema de gobernanza: una perspectiva de los grupos de presión”, en Maria del Carmen Pardo, Mauricio I. Dussauge L. y Guillermo M. Cejudo (eds.), Implementación de políticas públicas. Una antología, México, CIDE, pp. 247-282.

Capano, Gilberto, Andrea Pritoni y Giulia Vicentini (2017), "Policy instruments matter! How goverments choice of policy mix shapes higher education performance in Western Europe", en International Conference on Public Policy, Singapore.

CEPAL (2005), Indicadores de desempeño en el sector público, CEPAL (J. Bonnefoy y Armijo (coords.), Instituto Latinoamericano y del Caribe de Planificación Social (ILPES), Serie Manuales, recuperado de: <https:// repositorio.cepal.org/bitstream/handle/11362/5611/ S05900_es.pdf> [Consulta: junio de 2019].

Clark, Burton (1998), Creating entreprenurial universities: organisational pathways of tranformation, Oxford, Pergamon Press.

Clark, Burton (1983), The higher education system. academic organization in cross national perspective, Berkeley, University of California Press.

Boer, Harry y Jon File (2009), Higher education governance: reforms across Europe, Bruselas, ESMU.

De Vries, Wietse y Germán Álvarez-Mendiola (2012), "Reforms of university governance in Mexico: inducements for or impediments to change?", en Hans G. Schuetze, William Bruneau y Garnet Grosjean (eds.), University governance and reform. Policy, fads and experience in international perspective, Nueva York, Palgrave McMillan, pp. 125-146.

Easton, David (1985), "Political science in the United States”, International Political Science Review, vol. 6, núm.1 (The Future of the State), pp. 133-152.

El-Khawas, Elaine (2002), "Governance in US universities: aligning internal dynamics with todays needs", en A. M. Amaral, G. L. Jones y B. Karseth, Governing higher 
education: national perspectives on institutional governance, Dordrecht/Boston/London, Kluwer Academic Publishers, pp. 261-278.

Erwin, Cathleen, Amy Landry, Avery Livingstone y Ashley Dias (2019), "Effective governance and hospital boards revisited: reflections on 25 years of research", Medical Care Research and Review, vol. 76, núm.2, pp. 131-166.

Ganga-Contreras, Francisco, Juan Abello y Juan Quiroz (2014), "Gobernanza universitaria: una mirada histórica y conceptual", en F. Ganga, J. Abello y J. Quiroz, Gobernanza Universitaria: aproximaciones téoricas y empiricas, Santiago, Editorial CEDAC/Universidad de Los Lagos, pp. 11-20.

Ganga-Contreras, Francisco, Juan Quiroz y Paulo Fossati (2017), “Análisis sincrónico de la gobernanza universitaria: una mirada teórica a los años sesenta y setenta", Educación y Pesquisa, vol. 43, núm. 2, pp. 553-568.

Ganga-Contreras, Francisco y Patricio Viancos (2018), "Tipología de universidades: una propuesta a partir del rol del máximo directivo", Revista Dilemas Contemporáneos: Educación, Política y Valores, Año: V, núm. 2, pp. 1-18.

Ganga-Contreras, Francisco y Osman Nuñez (2018), "Gobernanza de las organizaciones: acercamiento conceptual a las instituciones de educación superior", Revista Espacios, vol. 39, núm. 17, pp. 1-14.

Hambrick, Donald, Axel Werder y Edward Zajac (2008), "New directions in corporate governance research", Organization Science, vol. 19, núm. 3, pp. 381-385.

Hardiman, Niamh (2012), "Governance and State Structures", en David Levi-Faur (ed.), The Oxford Handbook of Governance, Oxford, Oxford University Press, pp. 228-241.

Hernandez, Daniel, Mónica Orozc y Sirenia Vasquez (2005), La focalización como estrategia de política pública, México, Secretaría de Desarrollo Social.

Kent, Rollin (coord.) (2009), Las políticas de educación superior en México durante la modernización. Un análisis regional, México, ANUIES.

King, Roger (2015), "Institutional autonomy and accountability", en Jeroen Huisman, Harry de Boer, David D. Dill y Manuel Souto-Otero (eds.), The Palgrave
International Handbook of Higher Education Policy and Governance, Basingstoke, Palgrave Macmillan, pp. 485-505.

Lascoumes, Pierre y Patrick Le Galès (2014), Sociología de la acción pública, México, El Colegio de México.

Li, Shaomin, Seung Park y Rosey Bao (2019), "The transition from relation-based to rule-based governance in East Asia theories, evidence, and challenges", International fournal of Emerging Markets, vol. 14, núm. 1, pp. 171-186.

Maggio, Zachary (2011), "Exploring Burton Clark's triangle of coordination in the context between states and higher education systems", Nueva York, New York University (inédito).

McCormick, Alexander (2013), "Clasificación de las instituciones de educación superior: lecciones de la Clasificación Carnegie. Pensamiento educativo", Revista de Investigación Educacional Latinoamericana, vol. 50, núm. 1, pp. 65-75.

May, Peter (2018), "Reconsiderando los fracasos en la implementación: la perspectiva de los regímenes de políticas públicas", en María del Carmen Pardo, Mauricio I. Dussauge L. y Guillermo M. Cejudo (eds.), Implementación de políticas públicas. Una antología, México, CIDE, pp. 283-318.

Musselin, Christen y Stéphane Mignot-Gérard (2002), "The recent evolution of French universities", en A. M. Amaral, G. L. Jones y B. Karseth, Governing higher education: national perspectives on institutional governance, Dordrecht/Boston/London, Kluwer Academic Publishers.

North, Douglass (1993), Instituciones, cambio institucional y desempeño económico, México, Fondo de Cultura Económica.

OECD (2002), "Glosario de los principales términos sobre evaluación y gestión basada en resultados", recuperado de <https://www.oecd.org/dac/evaluation/2754804. pdf $>$ [Consulta: febrero de 2019].

Paradise, Catherine y Jean-Claude Thoenig (2017), En busca de la calidad académica, México, Fondo de Cultura Económica.

Pierre, Jon, (2012), "Governance and institutional flexibility", en David Levi-Faur (ed.), The Oxford 
Handbook of Governance, Oxford, Oxford University Press, pp. 187-200.

Porter, Michael (2002), Ventaja competitiva, México, Alay Ediciones.

Putnam, Robert (1993), Making democracy works: civic traditions in modern Italy, Princeton, Princeton University Press.

Rama, Claudio (2015), La universidad latinoamericana en la encrucïada de sus tendencias, Santa Cruz, Universidad Católica Boliviana "San Pablo".

Rama, Glaudio (2014), Los nuevos modelos universitarios en América Latina, Montevideo, Magro.

Reale, Emanuela y Emilia Primeri (2015), "Approaches to policy and governance in higher education", en Jeroen Huisman, Harry de Boer, David D. Dill y Manuel Souto-Otero (eds.), The Palgrave International Handbook of Higher Education Policy and Governance, Basingstoke, Palgrave Macmillan, pp. 20-37.

Rodríguez, Sebastián (2014), "El gobierno de las universidades: de la reflexión a la acción”, Bordón, número monográfico, vol. 66, núm. 1, enero-marzo, pp. 89-106.

Rosanvallon, Pierre (2007), La contrademocracia. La política en la era de la desconfianza, Buenos Aires, Manantial.

Salmi, Jamil (2009), El desafio de crear universidades de rango mundial, Washington, Banco Mundial.

Shattock, Michael (2014), "The context of 'modernising' reforms in university governance", en M. Shattock (ed.), International trends in university governance. Autonomy, self-government and the distribution of authority, Londres/ Nueva York, Routledge, pp. 1-14

Swianiewicz, Pawel (2000), "Institutional performance of local government administration", en J. Jabes Poland (ed.), Ten years of transition: prospects and challenges for the future of public administration, Budapest, Annual
Conference of NISPACEE, 13-15 april, pp. 162-186.

Teichler, Ulrich (1998), "The changing roles of the university and non-university sectors of higher education in Europe", European Review, vol. 6, núm. 4, pp. $475-487$.

Tight, Malcom (2015), "Research on higher education policy and institutional management", en Jeroen Huisman, Harry de Boer, David D. Dill y Manuel Souto-Otero (eds.), The Palgrave International Handbook of Higher Education Policy and Governance, Basingstoke, Palgrave Macmillan, pp. 176-191.

Van Vught, Frans and Harry De Boer (2015), "Governance models and policy instruments", en Jeroen Huisman, Harry de Boer, David D. Dill y Manuel Souto-Otero (eds.), The Palgrave International Handbook of Higher Education Policy and Governance, Basingstoke, Palgrave Macmillan, pp. 38-57.

Veiga, Amélia, António Magalhaes y Alberto Amaral (2015), "From collegial governance to boardism: reconfiguring governance in higher education", en Jeroen Huisman, Harry de Boer, David D. Dill y Manuel Souto-Otero (eds.), The Palgrave International Handbook of Higher Education Policy and Governance, Basingstoke, Palgrave Macmillan, pp. 398-416.

Wilson, Carter (2000), "Policy regimes and policy change", Journal of Public Policy, vol. 20, núm. 3, pp. 247-274.

Zgaga, Pavel (2012), "Reconsidering the EHEA principles: is there a "Bologna philosophy?", en A. S. Curaj, P. Scott, L. Vlasceanu y L. Wilson (eds.), European higher education at the crossroads: between the Bologna Process and national reforms (Part 1), pp. 17-32, Dordrecht, Springer, <https:// www.researchgate.net/publication/259561114_ Reconsidering_the_EHEA_Principles_Is_There_a_ Bologna_Philosophy> [Consulta: marzo de 2020].

Cómo citar este artículo: Acosta-Silva, Adrián, Francisco Ganga-Contreras y Claudio Rama-Vitale (2021), "Gobernanza universitaria: enfoques y alcances conceptuales", Revista Iberoamericana de Educación Superior (RIES), vol. XII, núm. 33, pp. 3-17, DoI: https://doi.org/10.22201/ iisue.20072872e.2021.33.854 [Consulta: fecha de última consulta]. 